\title{
CPD Questionnaire
}

\section{October 2016}

True (A) or false (B):

Cuban medical collaborations: Contextual and clinical challenges

1. With its strong emphasis on primary healthcare, the results of the study show that the Cuban medical curriculum was adequately preparing students for the South African context.

Remembering old partnerships: Networking as new medical schools within BoLeSwa countries

2. Prior to the establishment of the University of Botswana School of Medicine, Botswana had been placing students in more than 15 of their partner medical schools.

Tracking Master of Public Health graduates: Linking higher education and the labour market

3. As elsewhere in Africa, this study showed that public health graduates were mostly medical doctors.

Research supervision: Perceptions of postgraduate nursing students at a higher education institution in KwaZulu-Natal, South Africa

4. The literature shows a very weak association between the quality of supervision and the capacity of academic staff.

The electrocardiogram made (really) easy: Using small-group tutorials to teach electrocardiogram interpretation to final-year medical students

5. The need for an earlier introduction and more tutorials on electrocardiogram interpretation was one of the major findings of the study.

Effect of bedside teaching activities on patients' experiences at an Ethiopian hospital

6. Confidentiality was not raised as a concern by the patients included in this study.

Student feedback on an adapted appraisal model in resource-limited settings

7. Approximately half of the students who participated in the study felt uncomfortable talking about their personal problems with their appraiser.

The NOMA track module on nutrition, human rights and governance: Part 1. Perceptions held by Master's students

8. A highlight of the module for students was the opportunity to learn about the background to international rights in Norway.

The NOMA track module on nutrition, human rights and governance: Part 2. A transnational curriculum using a human rights-based approach to foster key competencies in nutrition professionals

9. The principles of the human rights-based approach emphasise participation, transparency, sustainability, and comprehensiveness.

Professional nurses' perception of their clinical teaching role at a rural hospital in Lesotho

10. The findings of this study demonstrate that skills development, critical thinking and clinical judgement in the clinical environment are important tasks that require training.
Allied health professional rural education: Stellenbosch University learners' experiences

11. Context, educators, and time were identified as the three core themes of professional education curricula.

Self-assessment of final-year undergraduate physiotherapy students' literature-searching behaviour, self-perceived knowledge of their own critical appraisal skills and evidence-based practice beliefs

12. Inadequate access to the internet and to medical literature was noted as a challenge by the students in this study.

The usefulness of a tool to assess reflection in a service-learning experience

13. Petersen and Osman (2013), cited in this article, suggest that reflection should start on a dialectical level and move towards a contextual holistic view of the situation.

Multidisciplinary leadership training for undergraduate health science students may improve Ugandan healthcare

14. Students who participated in the multidisciplinary leadership training programme showed minimal improvement in the competencies examined.

Exploring occupational therapy graduates' conceptualisations of occupational justice in practice: Curriculum implications

15. The challenges reported by the participants in the study concur with those identified by occupational therapists who practise in rural contexts and settings where the biomedical model dominates.

Burnout among paramedic students at a university in Johannesburg, South Africa

16. The prevalence of burnout among the cohort of students in this study was greater than that found in studies on medical students.

Registrars teaching undergraduate medical students: A pilot study at the University of Pretoria, South Africa

17. A review study quoted in this article reported that $20 \%$ of a registrar's time is spent on teaching undergraduate medical students.

An inferential comparison between the capabilities and achievements of 1st-year medical and nursing students at the University of the Free State, Bloemfontein, South Africa

18. The three main stressors identified by the authors of the study were academic stressors, clinical stressors and personal/social stressors.

Developing social accountability in 1st-year medical students: A case study from the Nelson R Mandela School of Medicine, Durban, South Africa

19. The nature of the service activities that groups undertake in the Making a Difference programme is decided by the course convenor in consultation with the student groups.

Beyond the lecture: Teaching for professional development

20. Panel members who participated in this study felt that a teacher's lack of knowledge can be used to motivate student learning.

A maximum of 3 CEUs will be awarded per correctly completed test.

The CPD programme for AJHPE is administered by Medical Practice Consulting

CPD questionnaires must be completed online at www.mpconsulting.co.za

After submission you can check the answers and print your certificate.

Questions may be answered up to 6 months after publication of each issue.

Accreditation number: MDB015/175/02/2016 